\title{
Unplanned hospital readmissions following congenital heart diseases surgery
}

\author{
Prevalence and predictors
}

Ahmad S. Azhar, MBBS, FRCPC.

\section{ABSTRACT}

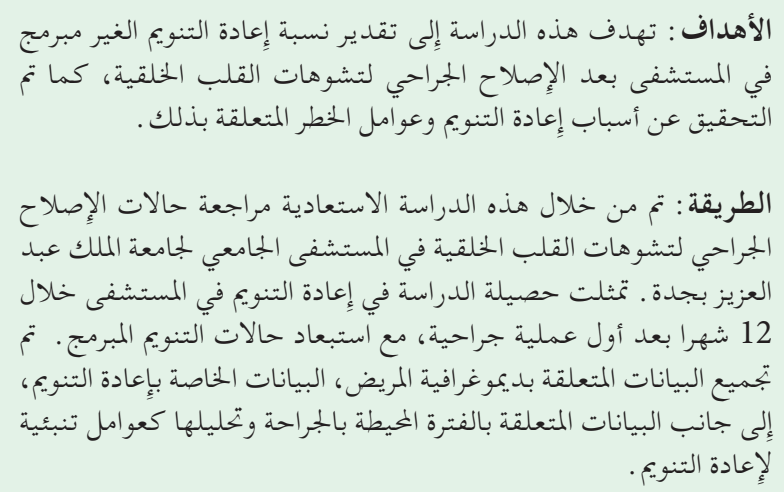

Objectives: To estimate the rate of unplanned hospital readmission following surgical repair of congenital heart defects (CHD) and investigate the related causes and risk factors.
Methods: A retrospective chart review of all the patients who underwent surgical repair of $\mathrm{CHD}$ at King Abdulaziz University Hospital, Jeddah, Saudi Arabia. The study outcome consisted of any hospital admission during the 12 months following the first reparative surgery. Exclusion criteria included planned admissions. Patients' demographic and readmission data as well as the perioperative data were collected and analyzed as factors and predictors of unplanned readmission

Results: After the exclusion of the deceased patients, a total of 189 patients were included. The readmission rate was $15.9 \%$ during a one-year period following surgery. There was a significant association between the probability of readmission and preoperative mechanical ventilation (MV) $(p<0.001)$, intraoperative complications $(p=0.025)$, prolonged postoperative length of stay (LOS) $\quad(p<0.001)$, early postoperative complication $(p=0.007)$, long postoperative MV stay, and drain tube stay $(p=0.011)$. Significant predictors of unplanned readmission included young age (1-12 months) and low weight at surgery $(<5 \mathrm{~kg})$, preoperative MV, intraoperative complications, postoperative LOS $\geq 10$ days, pediatric intensive care unit stay, MV stay, drain tube stay, infections, respiratory complication, and feeding problems. Only the preoperative MV and LOS $>10$ days were the independent risk factors.

Conclusion: Readmission rates were similar to those reported in other studies. Future studies are warranted to investigate suitable actions to alleviate the modifiable risk factors, such as postoperative complications.

Saudi Med J 2019; Vol. 40 (8): 802-809 doi: 10.15537/smj.2019.8.24405

From the Pediatric Department, Faculty of Medicine, King Abdulaziz University, Jeddah, Kingdom of Saudi Arabia.

Received 20th February 2019. Accepted 15th July 2019.

Address correspondence and reprint request to: Dr. Ahmad S. Azhar, Pediatric Department, Faculty of Medicine, King Abdulaziz University, Jeddah, Kingdom of Saudi Arabia. E-mail: azcardio@hotmail.com ORCID ID: https://orcid.org/0000-0002-0760-6086 
C ongenital heart disease (CHD) is the most common congenital disease among newborns in the world. ${ }^{1}$ Incidence estimates of CHD before the introduction of echocardiography ranged between 5 and 8 per 1,000 live births, while the current figures refer to approximately $8-12$ per 1,000 live births. ${ }^{2,3}$ In Saudi Arabia, CHD per 1,000 newborn infants was 2.1 where the data were extracted from stratified listing according to the population census, ${ }^{4} 5.4$ in Al-Qassim, ${ }^{5}$ and 7.4 in a study based on the national registry. ${ }^{6}$ The burden of CHD is predominant at the southwestern provinces and the northeast region of the Eastern province. ${ }^{6}$ The repair of heart congenital defects entails consuming high healthcare costs. Further, patients may experience hemodynamic residua or other types of complications later in their life, a matter which is more prominent in those with complicated anomalies. ${ }^{7}$ Therefore, it is plausible that patients with CHD are prone to readmission to the hospital after their initial procedures.

Hospital readmission may constitute a good reflect of the quality of the provided medical care and is associated with increased healthcare costs. ${ }^{8}$ Studies have shown that the readmission rate in children was $7 \%$ within 30 days of discharge ${ }^{9}$ In particular, following open-heart surgeries in the newborns, unplanned readmissions may have a significant influence on the emotional and financial aspects of the families. ${ }^{10}$ Accurate assessment of the readmission-related factors is of a significant implication in order to take the relevant actions to reduce the rates of unnecessary hospitalization and minimize health costs. However, only a small number of studies were performed to assess the prevalence rate of readmission after CHD surgeries in children. This study aimed at analyzing the incidence, causes, and risk factors associated with following CHD surgeries in children.

Methods. The current study is a retrospective chart review and includes patients who were subjected to primary surgical repair at the Pediatric Cardiology Unit, King Abdulaziz University Hospital (KAUH), Jeddah, Saudi Arabia between January 2013 and December 2016. It included all cases of children and adolescent (aged $\leq 14$ years) who underwent corrective or palliative surgery based on the type of CHD; and was approved

Disclosure. Authors have no conflict of interests, and the work was not supported or funded by any drug company. by the Institutional Review Board of KAUH. The study conformed to Helsinki Declaration.

The exclusion criteria included patients who were subjected to surgery after 14 years of age as well as secondary surgery cases.

A semi-structured form was used to collect data. The form included 5 divisions: i) baseline demographic and clinical factors (gender, age at surgery, CHD type, weight at surgery, presence of genetic syndrome, developmental delay, and so on); ii) preoperative factors as the need for mechanical ventilation (MV), preoperative length of stay (LOS)(days), baseline hemoglobin level (normal, low or high), and risk stratification using the risk adjusted classification for congenital heart surgery (RACHS) scale; iii) any intraoperative complications as injuries, bleeding, heart block, cardiac arrest; iv) postoperative data including pediatric intensive care unit (PICU) stay (days), drain tube stay (days), MV stay (days), and LOS from surgery to discharge and postoperative complications (cardiac, respiratory and renal complications, infections, feeding problems, and so on); v) readmission data including time from discharge to first readmission, cause, site (PICU or ward), readmission LOS, and number of readmission during the 12 months after surgery.

According to RACHS classification, cases were divided into 6 categories as previously described by Jenkins et al. ${ }^{11}$ Congenital heart disease cases were divided into 2 main groups of defects: group 1 including atrial septal defect, double outlet right ventricle, ventricular septal defect, tricuspid atresia patent ductus arteriosus; and group 2 including tetralogy of fallot, atrioventricular septal defect, transposition of the great arteries, pulmonary atresia, Total anomalous pulmonary venous return, coartaction of the aorta. The classification is based on postoperative length of stay findings that were described in a previous study involving the same population of patients. ${ }^{12}$

Statistical analysis. Data were analyzed using Statistical Package for Social Sciences version 21.0 for Windows (IBM SPSS Inc., Chicago, IL, USA). The primary study outcome was hospital readmission during the first year following surgical repair of CHD, and the related variable was analyzed as a binomial variable. Subsequently, participants were divided into 2 groups: readmitted and non-readmitted ones; groups were compared for different factors using Chi-square test or Fisher's exact test for categorical variables and independent t-test for continuous variables. Binary logistic regression was carried out to analyze predictors of readmission; univariate models were used to identify significant predictors and multivariate regression 
models were carried to analyze the significance of these predictors as independent risk factors. A $p$ value of $<0.05$ was considered to reject the null hypothesis.

Results. Out of 203 children and young adults who underwent surgical repair of CHD during the study period, 14 were excluded as they died before any readmission. The demographic and clinical characteristics of the 203 patients as well as their surgical outcomes were presented in a previously published paper that analyzed LOS. ${ }^{13}$ Consequently, 189 patients were included, 30 (15.9\%) were readmitted during the 12 months following congenital heart surgeries. Majority of readmission cases were in ward $(27 / 30,90.0 \%)$, occurred during the first month following discharge $(20 / 30,66.7 \%)$ and were motivated by infection (20/30, 66.7\%). Length of stay of readmitted patients was $<10$ days in $17 / 30(56.7 \%)$ of the cases (Table 1$)$.

Demographic and baseline clinical factors associated with unplanned hospital readmission. The percentage of readmissions was higher among younger age categories: $<30$ days $(17.6 \%)$ and $1-12$ months $(26.3 \%)$ versus $1-5$ years $(10.0 \%)$ and $>5$ years $(2.8 \%)(p=0.006)$; low weight at surgery $<5 \mathrm{~kg}(29.4 \%), 5-10 \mathrm{~kg}(18.3 \%)$ and $>10 \mathrm{~kg}(3.0 \%)(p<0.001)$; and patients with failure to thrive $(35.5 \%)$ versus those without $(12.0 \%)(p=0.001)$ (Table 2).

Perioperative factors of unplanned hospital readmissions. Readmission was associated with preoperative MV ( $40.7 \%$ versus $11.7 \%$; $p<0.001)$,

Table 1 - Readmissions during the 12 months following surgical repair of congenital heart defects ( $\mathrm{N}=30,15.9 \%$ of total participants).

\begin{tabular}{lcr}
\hline Parameter & Category & n (\%) \\
\hline \multirow{2}{*}{ Site } & PICU & $3(10.0)$ \\
& Ward & $27(90.0)$ \\
Time from discharge to & $<30$ days & $20(66.7)$ \\
$1^{\text {st }}$ readmission & $31-45$ days & $3(10.0)$ \\
& 46 days - 6 months & $4(13.3)$ \\
& $>6$ months - 1 year & $3(10.0)$ \\
Readmission LOS (days) & $<10$ & $17(56.7)$ \\
& $>=10$ & $13(43.3)$ \\
Cause of readmission & Infection & $20(66.7)$ \\
& Pleural effusion & $3(10.0)$ \\
& Pericardial effusion & $1(3.3)$ \\
Number of readmissions & 1 & $6(20.0)$ \\
during the 12 months & $2-3$ & $21(70.0)$ \\
following surgery* & & $5(16.7)$ \\
& & 4 \\
\end{tabular}

*For the same patient, PICU - pediatric intensive care unit, LOS - length of stay intraoperative complications $(41.7 \%$ versus $14.1 \%$; $p=0.025)$, extended postoperative LOS $(25.7 \%$ versus $4.5 \% ; p<0.001)$, and the occurrence of early postoperative complication $(21.1 \%$ versus $4.9 \%$; $p=0.005)$ by comparison to their counterparts. Intraoperative complications were reported in 12

Table 2 - Participants demographic and clinical characteristics and their correlation with postoperative length of hospital stay $(\mathrm{N}=189$, excluding 14 deaths).

\begin{tabular}{|c|c|c|c|c|}
\hline Parameter & Category & $\begin{array}{c}\text { No } \\
(\mathrm{n}=173)\end{array}$ & $\begin{array}{c}\text { Yes } \\
(\mathrm{n}=30)\end{array}$ & $P$-value \\
\hline \multicolumn{5}{|l|}{ Demographic data } \\
\hline \multirow{4}{*}{$\begin{array}{l}\text { Age at surgery } \\
\text { (category) }\end{array}$} & $<30$ days & $14(82.4)$ & $3(17.6)$ & \multirow{4}{*}{$0.006^{*}$} \\
\hline & $1-12$ months & $56(73.7)$ & $20(26.3)$ & \\
\hline & $1-5$ years & $54(90.0)$ & $6(10.0)$ & \\
\hline & $>5$ years & $35(97.2)$ & $1 \quad(2.8)$ & \\
\hline \multirow{2}{*}{ Gender } & Male & 79 (80.6) & 19 (19.4) & \multirow{2}{*}{0.170} \\
\hline & Female & $80(87.9)$ & $11(12.1)$ & \\
\hline \multirow[t]{2}{*}{ Nationality } & Non-Saudi & $144(83.2)$ & $29(16.8)$ & \multirow{2}{*}{$0.475^{\ddagger}$} \\
\hline & Saudi & $15(93.8)$ & $1 \quad(6.3)$ & \\
\hline \multicolumn{5}{|l|}{ Clinical data } \\
\hline \multirow{2}{*}{ Prematurity } & Yes & $2(84.4)$ & $29(15.6)$ & \multirow{2}{*}{$0.406^{\ddagger}$} \\
\hline & No & 157 (66.7) & $1(33.3)$ & \\
\hline \multirow{2}{*}{ Defect group } & Group 1 & $74(84.1)$ & $14(15.6)$ & \multirow{2}{*}{0.990} \\
\hline & Group 2 & $85(84.2)$ & $16(15.8)$ & \\
\hline \multirow{12}{*}{ Main defect } & VSD & $38(88.4)$ & $5(11.6)$ & \multirow{12}{*}{0.569} \\
\hline & TOF & $35(89.7)$ & $4(10.3)$ & \\
\hline & TGA & $25(80.6)$ & $6(19.4)$ & \\
\hline & AVSD & $12(75.0)$ & $4(25.0)$ & \\
\hline & ASD & $9(100)$ & 0 & \\
\hline & DORV & $5(71.4)$ & $2(28.6)$ & \\
\hline & Pulm. atresia & $5(100)$ & 0 & \\
\hline & Tricus. atresia & $4(66.7)$ & $2(33.3)$ & \\
\hline & TAPVC & $4(80.0)$ & $1(20.0)$ & \\
\hline & $\mathrm{CoA}$ & $4(80.0)$ & $1(20.0)$ & \\
\hline & PDA & $3(100)$ & 0 & \\
\hline & Other $^{\dagger}$ & $15(75.0)$ & $5(25.0)$ & \\
\hline \multirow{2}{*}{$\begin{array}{l}\text { Number of } \\
\text { defects }\end{array}$} & One defect & $132(83.5)$ & $26(16.5)$ & \multirow{2}{*}{$0.791^{\ddagger}$} \\
\hline & $\geq 2$ defects & $27(87.1)$ & $4(12.9)$ & \\
\hline \multirow{2}{*}{$\begin{array}{l}\text { Genetic } \\
\text { syndrome }\end{array}$} & None & $134(84.3)$ & $25(15.7)$ & \multirow{2}{*}{$1.000^{\ddagger}$} \\
\hline & Yes & $25(83.3)$ & $5(16.7)$ & \\
\hline \multirow{2}{*}{$\begin{array}{l}\text { Past heart } \\
\text { surgery }\end{array}$} & No & $142(84.5)$ & $26(15.5)$ & \multirow{2}{*}{$0.751^{\ddagger}$} \\
\hline & Yes & $17(81.0)$ & $4(19.0)$ & \\
\hline \multirow{3}{*}{$\begin{array}{l}\text { Weight at } \\
\text { surgery (kg) }\end{array}$} & $<5$ & $36(70.6)$ & $15(29.4)$ & \multirow{3}{*}{$<0.001^{*}$} \\
\hline & $5-10$ & $58(81.7)$ & $13(18.3)$ & \\
\hline & $>10$ & $65(97.0)$ & $2(3.0)$ & \\
\hline \multirow{2}{*}{ Failure to thrive } & No & $139(88.0)$ & $19(12.0)$ & \multirow{2}{*}{$0.001^{*}$} \\
\hline & Yes & $20(64.5)$ & $11(35.5)$ & \\
\hline $\begin{array}{l}\text { Developmental } \\
\text { delay }\end{array}$ & No & $141(85.5)$ & $24(14.5)$ & 0.190 \\
\hline \multicolumn{5}{|c|}{$\begin{array}{l}\text { Values are presented as frequencies and percentages }(\%) \text {, unless otherwise } \\
\text { specified. * *tatistically significant result }(p<0.05) \text {, }{ }^{\dagger} \text { other defects are } \\
\text { presented in a previous paper }{ }^{12},{ }^{\ddagger} \text { significance level calculated using Fisher's } \\
\text { exact test VSD - ventricular septal defect, TOF - tetralogy of fallot, } \\
\text { TGA - transposition of the great arteries, AVSD - atrioventricular septal } \\
\text { defect, ASD - atrial septal defect, DORV - double-outlet right ventricle, } \\
\text { TAPVC - total anomalous pulmonary venous return, CoA - coartaction } \\
\text { of the aorta, PDA - patent ductus arteriosus, Pulm. atresia - pulmonary } \\
\text { atresia, Tricus. atresia - tricuspid atresia }\end{array}$} \\
\hline
\end{tabular}


patients $(6.3 \%)$ and included 3 cases of cardiac arrest, 3 cases of injuries, 3 cases of bleeding, 2 cases of heart block, and one case of diaphragmatic paralysis. Further, long postoperative MV stay $(p=0.007)$ and drain tube stay $(p=0.011)$ were associated with higher percentage of readmissions (Table 3 ).

Predictors of unplanned readmission. In univariate regression models, age at surgery 1-12 months (odds ratio $[\mathrm{OR}]=12.50 ; p=0.016)$, low weight at surgery $(<5 \mathrm{~kg}: \mathrm{OR}=13.54, p=0.001 ; 5-10 \mathrm{~kg}: \mathrm{OR}=7.28$, $p=0.011)$, preoperative $\mathrm{MV}(\mathrm{OR}=5.17, p<0.001)$, as well as intraoperative complications $(\mathrm{OR}=4.34, p=0.019)$ were significant predictors of unplanned readmission. Postoperative predictors included postoperative LOS $\geq 10$ days $(\mathrm{OR}=7.28, \quad p<0.001)$, PICU stay $(\mathrm{OR}=1.07, p=0.001)$, MV stay $(\mathrm{OR}=1.14, p=0.002)$, drain tube stay $(\mathrm{OR}=1.05, \quad p=0.016)$, infections $(\mathrm{OR}=3.29, \quad p=0.006)$, respiratory complication $(\mathrm{OR}=4.32, p<0.001)$, and presence of feeding problems $(\mathrm{OR}=3.04, p=0.022)$ (Table 4). Based on significant predictors in univariate regression analysis, various multivariate models were tested; among these a 4-factor model showed preoperative $\mathrm{MV}(\mathrm{OR}=3.95, p=0.005)$ and postoperative $\mathrm{LOS}>10$ days $(\mathrm{OR}=3.60, p=0.038)$ to be the only independent risk factors of readmission (Table 5).

Discussion. The present study probed into unplanned hospital readmission following CHD first repair surgery and showed that one in 7 patients are readmitted in hospital during the 12 months of postoperative follow up; by exclusion of those who deceased during intervention or in early postoperative time. Further, this study highlighted 2 independent risk factors for unplanned readmission including preoperative MV and extended postoperative LOS (>10 days).

Unexpectedly, plausible parameters such as RACHS category, intraoperative complications, and the occurrence and number of postoperative complications were not demonstrated to be independently associated with unplanned hospital readmission; although, these showed to be statistically significant predictors in univariate regression models.

Despite the insufficient consensus about its exact quantifiable performance parameters, hospital readmission remains to be an essential element of the decision-making process for healthcare providers and policymakers who strive for delivering optimal quality care along with consumption of minimal resources. ${ }^{14,15}$ Readmission is basically linked to index hospitalization in general. During the past 2 decades, postoperative

Table 3 - Perioperative factors of unplanned hospital readmissions following surgical repair of congenital heart defects.

\begin{tabular}{|c|c|c|c|c|}
\hline Parameter & Category & $\begin{array}{c}\text { No } \\
(\mathrm{N}=173)\end{array}$ & $\begin{array}{c}\text { Yes } \\
(\mathrm{N}=30)\end{array}$ & $P$-value \\
\hline \multicolumn{5}{|l|}{ Preoperative data } \\
\hline \multirow{2}{*}{ Preoperative MV } & Yes & $143(88.3)$ & $19(11.7)$ & \multirow{2}{*}{$<0.001^{*}$} \\
\hline & No & $16(59.3)$ & $11(40.7)$ & \\
\hline \multirow{3}{*}{$\begin{array}{l}\text { Preoperative } \\
\text { hemoglobin level }\end{array}$} & Normal & $43(82.7)$ & $9(17.3)$ & \multirow{3}{*}{0.715} \\
\hline & Low & $91(83.5)$ & $18(16.5)$ & \\
\hline & High & $25(89.3)$ & $3(10.7)$ & \\
\hline \multirow{3}{*}{$\begin{array}{l}\text { Preoperative } \\
\text { LOS (days) }\end{array}$} & $<2$ & $81(89.0)$ & $10(11.0)$ & \multirow{3}{*}{0.100} \\
\hline & $2-5$ & $38(84.4)$ & $7(15.6)$ & \\
\hline & $>5$ & $40(75.5)$ & $13(24.5)$ & \\
\hline \multirow[t]{6}{*}{ RACHS category } & 1 & $11(100)$ & 0 & \multirow{6}{*}{$0.011^{*}$} \\
\hline & 2 & $102(84.3)$ & $19(15.7)$ & \\
\hline & 3 & $35(85.4)$ & $6(14.6)$ & \\
\hline & 4 & $8(80.0)$ & $2(20.0)$ & \\
\hline & 5 & 0 & 0 & \\
\hline & 6 & 0 & $2(100)$ & \\
\hline \multicolumn{5}{|l|}{ Intraoperative data } \\
\hline Surgical & No & $152(85.9)$ & $25(14.1)$ & \multirow{2}{*}{$0.025^{*}$} \\
\hline complications & Yes & $7(58.3)$ & $5(41.7)$ & \\
\hline \multicolumn{5}{|l|}{ Postoperative data } \\
\hline \multirow{3}{*}{$\begin{array}{l}\text { Postoperative LOS } \\
\text { (days) }\end{array}$} & $<10$ & $84(95.5)$ & 4 (4.5) & \multirow{3}{*}{$<0.001^{*+}$} \\
\hline & $10+$ & $75(74.3)$ & $26(25.7)$ & \\
\hline & $<2$ & $11(100)$ & 0 & \\
\hline \multirow{4}{*}{ PICU stay (days) } & $2-5$ & $89(90.8)$ & $9(8.2)$ & \multirow{4}{*}{$<0.001^{*}$} \\
\hline & $6-10$ & $40(83.3)$ & $8(16.7)$ & \\
\hline & $>10$ & $19(59.4)$ & $13(40.6)$ & \\
\hline & $<1$ & $71(91.0)$ & $7(9.0)$ & \\
\hline \multirow{4}{*}{$\begin{array}{l}\text { Mechanical } \\
\text { ventilation (days) }\end{array}$} & $1-5$ & $74(84.1)$ & $14(15.9)$ & \multirow{3}{*}{$0.007^{*}$} \\
\hline & $6-10$ & $9(60.0)$ & $6(40.0)$ & \\
\hline & $>10$ & $5(62.5)$ & $3(37.5)$ & \\
\hline & $<3$ & $5(100)$ & 0 & \multirow{5}{*}{$0.011^{*}$} \\
\hline \multirow{4}{*}{$\begin{array}{l}\text { Drain tube stay } \\
\text { (days) }\end{array}$} & $3-5$ & $80(93.0)$ & $6(7.0)$ & \\
\hline & $6-10$ & $39(76.5)$ & $12(23.5)$ & \\
\hline & $>10$ & $32(72.7)$ & $12(27.3)$ & \\
\hline & No drain & $3(100)$ & 0 & \\
\hline
\end{tabular}

Outcomes and complications

\begin{tabular}{|c|c|c|c|c|}
\hline Any complication & $\begin{array}{c}\text { None } \\
1 \text { or more }\end{array}$ & $\begin{array}{r}58(95.1) \\
101(78.9)\end{array}$ & $\begin{array}{r}3(4.9) \\
27(21.1)\end{array}$ & $0.005^{* \dagger}$ \\
\hline \multirow{2}{*}{ Infection } & No & $93(91.2)$ & $9(8.8)$ & \multirow{2}{*}{$0.004^{*}$} \\
\hline & Yes & $66(75.9)$ & $21(24.1)$ & \\
\hline \multirow{2}{*}{$\begin{array}{l}\text { Cardiac } \\
\text { complications }\end{array}$} & No & $129(84.3)$ & $24(15.7)$ & \multirow{2}{*}{0.885} \\
\hline & Yes & $30(83.3)$ & $6(16.7)$ & \\
\hline \multirow{2}{*}{$\begin{array}{l}\text { Feeding } \\
\text { complications }\end{array}$} & No & $142(86.6)$ & $22(3.4)$ & \multirow{2}{*}{$0.018^{*}$} \\
\hline & Yes & $17(68.0)$ & $8(32.0)$ & \\
\hline \multirow{2}{*}{$\begin{array}{l}\text { Respiratory } \\
\text { complication }\end{array}$} & No & $108(92.3)$ & $9(7.7)$ & \multirow{2}{*}{$<0.001^{*}$} \\
\hline & Yes & $51(70.8)$ & $21(29.2)$ & \\
\hline \multirow{2}{*}{ Transfusion } & No & $140(83.8)$ & $27(16.2)$ & \multirow{2}{*}{$1.000^{\dagger}$} \\
\hline & Yes & $19(86.4)$ & $3(13.6)$ & \\
\hline \multirow{5}{*}{$\begin{array}{l}\text { Number of } \\
\text { complications }\end{array}$} & 0 & $58(95.1)$ & $3(4.9)$ & \multirow{5}{*}{$<0.000^{*}$} \\
\hline & 1 & $53(88.3)$ & $7(11.7)$ & \\
\hline & 2 & $27(67.5)$ & $13(32.5)$ & \\
\hline & 3 & $14(87.5)$ & $2(12.5)$ & \\
\hline & $4+$ & $7(58.3)$ & $5(41.7)$ & \\
\hline \multirow{2}{*}{ Revision surgery } & No & $158(84.9)$ & $28(15.1)$ & \multirow{2}{*}{$0.066^{\dagger}$} \\
\hline & Yes & $1(33.3)$ & $2(66.7)$ & \\
\hline $\begin{array}{l}\text { Values are frequ } \\
\text { *statistically signif } \\
\text { Fisher's exact te }\end{array}$ & $s$ and per & $\begin{array}{l}\text { age (\%), unl } \\
\text { 5), 'significa } \\
\text { stay, MV - }\end{array}$ & $\begin{array}{l}\text { otherwise } \\
\text { level calcu } \\
\text { chanical ve }\end{array}$ & $\begin{array}{l}\text { ecified, } \\
\text { ted using } \\
\text { ilation }\end{array}$ \\
\hline
\end{tabular}


Table 4 - Perioperative factors of unplanned hospital readmissions following surgical repair of congenital heart defects.

\begin{tabular}{|c|c|c|c|c|c|}
\hline Predictor & Category/value & OR & \multicolumn{2}{|c|}{$95 \% \mathrm{CI}$} & $P$-value \\
\hline \multicolumn{6}{|l|}{ Demographic predictors } \\
\hline \multirow{4}{*}{ Age at surgery (category) } & $<30$ days & 7.50 & 0.72 & 78.37 & 0.092 \\
\hline & 1-12 months & 12.50 & 1.61 & 97.32 & $0.016^{*}$ \\
\hline & $1-5$ years & 3.89 & 0.45 & 33.70 & 0.218 \\
\hline & $>5$ years & (ref) & - & - & $0.019^{*}$ \\
\hline \multicolumn{6}{|l|}{ Baseline clinical predictors } \\
\hline & $<5$ & 13.54 & 2.93 & 62.57 & $0.001^{*}$ \\
\hline \multirow[t]{2}{*}{ Weight at surgery $(\mathrm{kg})$} & $5-10$ & 7.28 & 1.58 & 33.65 & $0.011^{*}$ \\
\hline & $>10$ & (ref) & - & - & $0.003^{*}$ \\
\hline \multicolumn{6}{|l|}{ Preoperative predictors } \\
\hline Mechanical ventilation & Yes & 5.17 & 2.09 & 12.79 & $<0.001^{*}$ \\
\hline \multirow{2}{*}{ RACHS category } & Low $(1-3)$ & (ref) & - & - & - \\
\hline & High (4-6) & 2.96 & 0.83 & 10.57 & 0.095 \\
\hline Intraoperative complications & Yes & 4.34 & 1.28 & 14.76 & $0.019^{*}$ \\
\hline \multicolumn{6}{|l|}{ Postoperative predictors } \\
\hline Postop LOS & (Days) & 1.03 & 1.01 & 1.06 & $0.003^{*}$ \\
\hline Postop LOS & $10+$ days & 7.28 & 2.43 & 21.82 & $<.001^{*}$ \\
\hline PICU stay & (Days) & 1.07 & 1.03 & 1.12 & $0.001^{*}$ \\
\hline MV stay & (Days) & 1.14 & 1.05 & 1.24 & $0.002^{*}$ \\
\hline Drain tube stay & (Days) & 1.05 & 1.01 & 1.10 & $0.016^{*}$ \\
\hline Infectious complication & Yes & 3.29 & 1.42 & 7.63 & $0.006^{*}$ \\
\hline Respiratory complication & Yes & 4.32 & 1.92 & 0.73 & $<0.001^{*}$ \\
\hline Diet/feeding problem & Yes & 3.04 & 1.17 & 7.88 & $0.022^{*}$ \\
\hline Any complication & Yes & 5.17 & 1.50 & 17.78 & $0.009^{*}$ \\
\hline
\end{tabular}

outcomes have been substantially improved in regards to mortality. ${ }^{16,17}$ Focusing on the congenital heart surgeries, several attempts have been established to reduce hospital LOS and the associated cost, such as employing a critical pathway ${ }^{18}$ or fast-track extubation, ${ }^{19}$ yielding promising outcomes. However, readmissions that may remain high in CHD patients should drive similar attention, as they yield comparable burden in term of morbidity and health expenditure.

In the present study, the rate of readmission was $10.5 \%$ within 30 days and $15.9 \%$ after one year of discharge following the congenital heart surgeries. The majority of studies from literature focused on the 30-day time frame. For example, a similar figure was reported in a previous study, ${ }^{20}$ where $10.8 \%$ of children with CHD in Atlanta were readmitted after their corrective surgeries. This was also typically consistent with a $10.5 \%$ readmission rate as per results of a multiinstitutional analysis in the United States. ${ }^{21}$ A slightly lower rate $(9.7 \%)$ was reported among the children who underwent elective cardiac surgery procedures. ${ }^{22}$ On the other hand, in a large population-based study in Canada, the rate of readmission within 30 days was larger $(14 \%)$ in children aged $0-17$ years during a 15 -year period. ${ }^{10}$ Readmission rates may differ by type of CHD and or procedure; for example, Mackie et $\mathrm{al}^{23}$ found that the readmission rates were 3.8\% following arterial switch procedures, while $11.4 \%$ readmissions were reported after Norwood procedures. In general, the readmission rates in KAUH were relatively similar to those of other studies. However, readmission to other hospitals may interfere the true rates, since the patient with an urgent intervening illness (comorbid with CHD) may be readmitted to another hospital closer to his/her home.

Preoperative MV and postoperative LOS can be considered as nonspecific parameters that may be independently associated to other more clinically significant factors such as RACHS category, type of $\mathrm{CHD}$, type of surgery; however, investigating these associations was beyond the scope of this study. Consistent with our findings, Kogon et $\mathrm{al}^{20}$ found also that MV was one of the associated preoperative factors with the likelihood of readmission, which was in agreement with Saharan et al. ${ }^{13}$ Actually, the preoperative criteria of the patients reflect the severity 
Table 5 - Independent risk factors of readmission following surgical repair of congenital heart defects (multivariate binary logistic regression).

\begin{tabular}{|c|c|c|c|c|c|}
\hline \multirow{2}{*}{$\begin{array}{l}\text { Model/Predictor } \\
\text { Model } 1\end{array}$} & \multirow[t]{2}{*}{ Category } & \multirow[t]{2}{*}{ OR } & \multicolumn{2}{|c|}{$95 \% \mathrm{CI}$} & \multirow[t]{2}{*}{$P$-value } \\
\hline & & & & & \\
\hline \multicolumn{6}{|l|}{ Demographic predictors } \\
\hline \multirow[t]{4}{*}{ Age at surgery (category) } & $<30$ days & 0.47 & 0.01 & 17.67 & 0.683 \\
\hline & $1-12$ months & 2.77 & 0.12 & 66.58 & 0.530 \\
\hline & $1-5$ years & 1.64 & 0.09 & 31.44 & 0.741 \\
\hline & $>5$ years & (ref) & - & - & - \\
\hline \multicolumn{6}{|l|}{ Baseline clinical predictors } \\
\hline & $<5$ & 3.62 & 0.24 & 53.65 & 0.349 \\
\hline \multirow[t]{2}{*}{ Weight at surgery (kg) } & $5-10$ & 4.22 & 0.42 & 42.33 & 0.221 \\
\hline & $>10$ & (ref) & - & - & - \\
\hline \multicolumn{6}{|l|}{ Preoperative predictors } \\
\hline Mechanical ventilation & Yes & 2.95 & 0.80 & 10.82 & 0.103 \\
\hline $\begin{array}{l}\text { Intraoperative } \\
\text { complications }\end{array}$ & Yes & 2.28 & 0.49 & 10.61 & 0.293 \\
\hline \multicolumn{6}{|l|}{ Postoperative predictors } \\
\hline Postop LOS & (days) & 0.99 & 0.95 & 1.02 & 0.466 \\
\hline Postop LOS & $10+$ days & 3.30 & 0.89 & 12.25 & 0.074 \\
\hline PICU stay & (Days) & 1.02 & 0.93 & 1.11 & 0.705 \\
\hline MV stay & (Days) & 1.02 & 0.86 & 1.21 & 0.810 \\
\hline Drain tube stay & (Days) & 1.03 & 0.96 & 1.10 & 0.395 \\
\hline Infectious complication & Yes & 1.27 & 0.38 & 4.18 & 0.697 \\
\hline Respiratory complication & Yes & 2.06 & 0.65 & 6.55 & 0.219 \\
\hline Diet/feeding problem & Yes & 1.80 & 0.49 & 6.63 & 0.378 \\
\hline Any complication & Yes & 1.05 & 0.17 & 6.41 & 0.959 \\
\hline \multicolumn{6}{|l|}{ Model 2} \\
\hline \multicolumn{6}{|l|}{ Baseline clinical predictors } \\
\hline & $<5$ & 4.44 & 0.76 & 26.12 & 0.099 \\
\hline \multirow[t]{2}{*}{ Weight at surgery (kg) } & $5-10$ & 6.59 & 1.32 & 32.98 & $0.022^{*}$ \\
\hline & $>10$ & (ref) & - & - & - \\
\hline \multicolumn{6}{|l|}{ Preoperative predictors } \\
\hline Mechanical ventilation & Yes & 2.94 & 0.87 & 9.94 & 0.083 \\
\hline $\begin{array}{l}\text { Intraoperative } \\
\text { complications }\end{array}$ & Yes & 1.57 & 0.36 & 6.83 & 0.550 \\
\hline \multicolumn{6}{|l|}{ Postoperative predictors } \\
\hline Postop lenght of stay & $10+$ days & 3.29 & 0.89 & 12.17 & 0.074 \\
\hline PICU stay & (Days) & 0.99 & 0.91 & 1.07 & 0.777 \\
\hline MV stay & (Days) & 1.05 & 0.90 & 1.23 & 0.517 \\
\hline Drain tube stay & (Days) & 1.01 & 0.96 & 1.07 & 0.701 \\
\hline Infectious complication & Yes & 1.19 & 0.38 & 3.80 & 0.764 \\
\hline Respiratory complication & Yes & 1.87 & 0.62 & 5.62 & 0.265 \\
\hline Diet/feeding problem & Yes & 1.48 & 0.44 & 4.95 & 0.522 \\
\hline Any complication & Yes & 1.11 & 0.20 & 6.32 & 0.904 \\
\hline \multicolumn{6}{|l|}{ Model 3} \\
\hline Baseline clinical predictors & & & & & \\
\hline & $<5$ & 4.32 & .78 & 24.08 & 0.095 \\
\hline Weight at surgery $(\mathrm{kg})$ & 5-10 & 6.23 & 1.28 & 30.31 & $0.023^{*}$ \\
\hline & $>10$ & (ref) & - & - & - \\
\hline Preoperative predictors & & & & & \\
\hline Mechanical ventilation & Yes & 3.11 & 1.00 & 9.65 & $0.049^{*}$ \\
\hline Postoperative predictors & & & & & \\
\hline Postop length of stay & $10+$ days & 3.90 & 1.14 & 13.34 & $0.030^{*}$ \\
\hline MV stay & (Days) & 1.05 & .97 & 1.14 & 0.241 \\
\hline Respiratory complication & Yes & 2.04 & .76 & 5.46 & 0.156 \\
\hline Model 4 & & & & & \\
\hline Preoperative predictors & & & & & \\
\hline Mechanical ventilation & Yes & 3.95 & 1.50 & 10.40 & $0.005^{*}$ \\
\hline Postoperative predictors & & & & & \\
\hline Postop length of stay & $10+$ days & 3.60 & 1.07 & 12.07 & $0.038^{*}$ \\
\hline MV stay & (Days) & 2.02 & 0.77 & 5.26 & 0.152 \\
\hline Respiratory complication & Yes & 1.07 & 0.98 & 1.16 & 0.112 \\
\hline
\end{tabular}

of their condition, where the need for longer MV associated with higher RACHS category indicated an increased burden of CHD. This in turn would require readmission following surgeries as the patient might experience significant hemodynamic instability and risk of hospital-acquired infection. ${ }^{24}$ Indeed, reports have shown that the patients with a complex CHD had a 2.3-4.1 greater risk of readmission after the index hospitalization. ${ }^{25}$

Total postoperative LOS for more than 10 days and PICU LOS were included as independent risk factors of readmission in the present study. This finding is consistently reported in most of the studies. ${ }^{10,13,20,23}$ In addition, Smith et $\mathrm{al}^{21}$ have shown that the overall hospital LOS and PICU LOS were approximately 2 -fold longer in the readmitted CHD patients. This was in agreement with the findings of Kogon et $\mathrm{al}^{20}{ }^{20}$ where the total postoperative LOS for more than or the same period was an independent predictor of readmission (relative risk [RR] 4.24, 95\% CI 2.26 to 7.96, $p<0.0001$ ) along with preoperative failure to thrive and the Hispanic ethnicity. Actually, the prolonged LOS is potentially explained by greater complicated conditions along with the lack of support in the outpatient setting. ${ }^{25}$ Further, the high rates of postoperative infection may represent a major contributor in extending the LOS as demonstrated in our previous study. ${ }^{12}$ In addition to increasing the liability to readmission, prolonged LOS in the hospital would consume a great amount of inpatient resources that could be originally prevented if adequately handled.

Although not being an independent risk factor, the occurrence of any postoperative complication was associated with 5.17 odd probability of readmission during the 12 months following surgery; with great significance for respiratory complications, infections and diet/feeding problems. However, this study did not explore the relationship between postoperative complications and the causes of readmission; reports from literature highlight the high prevalence of respiratory problems and infections in readmission among CHD patients. In the studies of Kogon et $\mathrm{al}^{20}$ and Saharan et $\mathrm{al},{ }^{13}$ pleural effusion was the leading cause of readmission following CHD surgeries, while the respiratory causes in general were the main etiologies of readmission in a study by Smith et al. ${ }^{21}$ Drainage tube stay was another factor that was statistically associated to readmission after $\mathrm{CHD}$ repair without being an independent risk factor. Pleural effusion continues to be real problem for the patients during the postoperative period and, if it persists for long time, it will cause a significant morbidity with prolongation of LOS. In 
our experience, a significant number of complications of Fontan surgery that caused readmission were related protein loosing enteropathy PLE and lymphatic dysfunctions. The exact reason for postoperative pleural effusion has not been clearly identified although some evidences have shown that the increase in pulmonary artery pressure, reduction of the ventricular function, and prolonged MV contribute to longer drainage tube stay. ${ }^{26,27}$ It is worthy to note that Glenn and Fontan procedures are the most common procedures wherein pleural effusion is observed ${ }^{13}$ despite the successful approach to reduce the overall drainage tube stay by a recent study by Pike et al. ${ }^{28}$

Similarly, infection represented one of the most prevalent cause of readmission in CHD patients. A recent study including 16 developing countries revealed that postoperative infection contributed significantly to morbidity and mortality after CHD surgery, leading to longer duration of postoperative MV and longer LOS in the PICU. ${ }^{29}$ Additionally, the risk of infection would be substantially increased in younger cases. Infants were more likely to be readmitted independent of the severity of their condition. ${ }^{30}$ This may be explained by their low physiologic reserve that might render a great risk of acquiring viral infection. From another perspective, Mackie et $\mathrm{a}^{10}$ suggested that there may be a less tendency of the physicians to hospitalize infants upon the incidence of complications when compared to older children.

The aforementioned observations should be interpreted within the boundaries of the study limitations; such as the retrospective design that generally entails considerable bias and lack of some key variables, which hinder adequate analysis of independent risk factors and confounders and their effect on the postoperative outcomes. Moreover, small sample size reduced the statistical power of some analysis, notably subgroup analysis by type of procedure, type of CHD, so on and their effect on readmission rate. Another important limiting factor is that some patients could not be included as they may be readmitted to other hospitals after their index hospitalization at KAUH. It is possible also that home nursing care and clinic visits following the surgery may be of a significant role that should be born in mind. Finally, available data did not enable investigating further factors such as the surgical proficiency of the surgeons, which may affect the surgical outcomes and subsequent complications and readmission.

In conclusion, approximately one in $7 \mathrm{CHD}$ patients are re-hospitalized during the 12 months following first repair surgery, and this rate is similar to that reported in other studies. Re-hospitalization was independently predicted by preoperative MV and extended postoperative LOS ( $>10$ days), both being probably linked to other more specific, modifiable factors that should be identified to reduce the rate of readmission and the related morbidity and costs. Other significant factors associated with re-hospitalization that were identified in this study; among these younger age, low weight at surgery, and preoperative MV, in addition to intra- and postoperative complications, drain tube stay, and presence of feeding problems. It is therefore recommended to take the suitable actions in order to control the modifiable risk factors such as infections as well as considering the possible role of surgical expertise in preventing the postoperative complications. This would ultimately serve a promising approach to reduce burden with to health and financial aspects.

Acknowledgment. The author acknowledges the valuable contribution of the Pediatric Cardiology Center of Excellence, of King Abdulaziz University Hospital, Jeddah, Saudi Arabia in sharing the data of congenital heart diseases between January 2013 to December 2016.

\section{References}

1. Fahed AC, Gelb BD, Seidman J, Seidman CE. Genetics of congenital heart disease: the glass half empty. Circ Res 2013; 112: 707-720.

2. Wren C. The epidemiology of cardiovascular malformations. In: Moller J, Hoffman J, Benson D, van Hare G, Wren C, editors. Pediatric Cardiovascular Medicine. Oxford (UK): Wiley-Blackwell; 2012. p. 268-275.

3. Hoffman JI. The global burden of congenital heart disease. Cardiovasc J Afr 2013; 24: 141-145.

4. Alqurashi M, El Mouzan M, Al Herbish A, Al Salloum A, Al Omer A. Symptomatic congenital heart disease in the Saudi Children and Adolescents Project. Ann Saudi Med 2007; 27: 442-444.

5. Al-Mesned A, Al Akhfash AA, Sayed M. Incidence of severe congenital heart disease at the province of Al-Qassim, Saudi Arabia. Congenit Heart Dis 2012; 7: 277-282.

6. Greer W, Sandridge A, Al-Menieir M, Al Rowais A. Geographical distribution of congenital heart defects in Saudi Arabia. Ann Saudi Med 2005; 25: 63-69.

7. Guleserian KJ. Adult congenital heart disease: surgical advances and options. Prog Cardiovasc Dis 2011; 53: 254-264.

8. Redžek A, Mironicki M, Gvozdenović A, Petrović M, ČemerlićAđić N, Ilić A, et al. Predictors for hospital readmission after cardiac surgery. J Card Surg 2015; 30: 1-6.

9. Berry JG, Toomey SL, Zaslavsky AM, Jha AK, Nakamura MM, Klein DJ, et al. Pediatric readmission prevalence and variability across hospitals. Jama 2013; 309: 372-380.

10. Mackie AS, Ionescu-Ittu R, Pilote L, Rahme E, Marelli AJ. Hospital readmissions in children with congenital heart disease: a population-based study. Am Heart J 2008; 155 : 577-584. 
11. Jenkins KJ, Gauvreau K, Newburger JW, Spray TL, Moller JH, Iezzoni LI. Consensus-based method for risk adjustment for surgery for congenital heart disease. J Thorac Cardiovasc Surg 2002; 123: 110-118.

12. Azhar AS, Aljefri HM. Predictors of Extended Length of Hospital Stay Following Surgical Repair of Congenital Heart Diseases. Pediatr Cardiol 2018; 39:1688-1699.

13. Saharan S, Legg AT, Armsby LB, Zubair MM, Reed RD, Langley SM. Causes of readmission after operation for congenital heart disease. Ann Thorac Surg 2014; 98: 1667-1673.

14. Billings J, Dixon J, Mijanovich T, Wennberg D. Case finding for patients at risk of readmission to hospital: development of algorithm to identify high risk patients. BMJ 2006; 333: 327.

15. Jencks SF, Brock JE. Hospital accountability and population health: lessons from measuring readmission rates. Ann Intern Med 2013; 159: 629-630.

16. Eghtesady P, Brar AK, Hall M. Prioritizing quality improvement in pediatric cardiac surgery. J Thorac Cardiovasc Surg 2013; 145: 631-640.

17. Williams DL, Gelijns AC, Moskowitz AJ, Weinberg AD, Ng $\mathrm{JH}$, Crawford E, et al. Hypoplastic left heart syndrome: valuing the survival. J Thorac Cardiovasc Surg 2000; 119: 720-731.

18. Turley K, Tyndall M, Roge C, Cooper M, Turley K, Applebaum $\mathrm{M}$, et al. Critical pathway methodology: effectiveness in congenital heart surgery. Ann Thorac Surg 1994; 58: 57-65.

19. Akhtar MI, Hamid M, Minai F, Wali AR. Safety profile of fast-track extubation in pediatric congenital heart disease surgery patients in a tertiary care hospital of a developing country: an observational prospective study. J Anaesthesiol Clin Pharmacol 2014; 30: 355-359.

20. Kogon B, Jain A, Oster M, Woodall K, Kanter K, Kirshbom P. Risk factors associated with readmission after pediatric cardiothoracic surgery. Ann Thorac Surg 2012; 94: 865-873.

21. Smith AH, Doyle TP, Mettler BA, Bichell DP, Gay JC. Identifying predictors of hospital readmission following congenital heart surgery through analysis of a multiinstitutional administrative database. Congenit Heart Dis 2015; 10: 142-152.
22. Vricella LA, Dearani JA, Gundry SR, Razzouk AJ, Brauer SD, Bailey LL. Ultra fast track in elective congenital cardiac surgery. Ann Thorac Surg 2000; 69: 865-871.

23. Mackie AS, Gauvreau K, Newburger JW, Mayer JE, Erickson LC. Risk factors for readmission after neonatal cardiac surgery. Ann Thorac Surg 2004; 78: 1972-1978.

24. Gupta P, Rettiganti M, Gossett JM, Yeh JC, Jeffries HE, Rice TB, et al. Risk factors for mechanical ventilation and reintubation after pediatric heart surgery. J Thorac Cardiovasc Surg 2016; 151: 451-458.

25. Islam S, Yasui Y, Kaul P, Mackie AS. Hospital readmission of patients with congenital heart disease in Canada. Can J Cardiol 2016; 32: 987.

26. Mascio CE, Wayment M, Colaizy TT, Mahoney LT, Burkhart HM. The modified Fontan procedure and prolonged pleural effusions. Am Surg 2009; 75: 175-177.

27. Border WL, Syed AU, Michelfelder EC, Khoury P, Uzark KC, Manning $\mathrm{PB}$, et al. Impaired systemic ventricular relaxation affects postoperative short-term outcome in Fontan patients. $J$ Thorac Cardiovasc Surg 2003; 126: 1760-1764.

28. Pike NA, Okuhara CA, Toyama J, Gross BP, Wells WJ, Starnes VA. Reduced pleural drainage, length of stay, and readmissions using a modified Fontan management protocol. J Thorac Cardiovasc Surg 2015; 150: 481-487.

29. Sen AC, Morrow DF, Balachandran R, Du X, Gauvreau K, Jagannath BR, et al. Postoperative infection in developing world congenital heart surgery programs: Data from the International Quality Improvement Collaborative. Circ Cardiovasc Qual Outcomes 2017; 10: e002935.

30. Björ O, Bråbäck L. A retrospective population based trend analysis on hospital admissions for lower respiratory illness among Swedish children from 1987 to 2000. BMC Public Health 2003; 3: 22. 